\title{
Peripheral and Tissue Lymphocytes as Predictors of Pathological Response in Locally Advanced Rectal Cancer Post Neoadjuvant Chemoradiotherapy
}

\author{
Shereen El Shorbagy', Ola M. Elfarargy ${ }^{1 *}$, Reham A. Salem², Amina M. Elnaggar ${ }^{3}$, Ola A. Harb4, \\ Abeer M. Abdelbary4, Hassan R. Ashour ${ }^{5}$, Loay M. Gertallah ${ }^{5}$ \\ ${ }^{1}$ Medical Oncology Department, Faculty of Medicine, Zagazig University, Zagazig, Egypt \\ ${ }^{2}$ Clinical Oncology \& Nuclear Medicine Department, Faculty of Medicine, Zagazig University, Zagazig, Egypt \\ ${ }^{3}$ Clinical Pathology Department, Faculty of Medicine, Zagazig University, Zagazig, Egypt \\ ${ }^{4}$ Pathology Department, Faculty of Medicine, Zagazig University, Zagazig, Egypt \\ ${ }^{5}$ General Surgery Department, Faculty of Medicine, Zagazig University, Zagazig, Egypt \\ Email: *elfarargyola@yahoo.com
}

How to cite this paper: El Shorbagy, S., Elfarargy, O.M., Salem, R.A., Elnaggar, A.M., Harb, O.A., Abdelbary, A.M., Ashour, H.R. and Gertallah, L.M. (2017) Peripheral and Tissue Lymphocytes as Predictors of Pathological Response in Locally Advanced Rectal Cancer Post Neoadjuvant Chemoradiotherapy. Journal of Cancer Therapy, 8, 250267.

https://doi.org/10.4236/jct.2017.83021

Received: January 28, 2017

Accepted: March 19, 2017

Published: March 22, 2017

Copyright ( 92017 by authors and Scientific Research Publishing Inc. This work is licensed under the Creative Commons Attribution International License (CC BY 4.0).

http://creativecommons.org/licenses/by/4.0/ (c) (i) Open Access

\begin{abstract}
Background: Tailoring therapy is the target in the management of any cancer; if factors which can predict response to treatment are identified, we can individualize treatment. Locally advanced rectal cancer studies reported that tumor microenvironment and host immune response played roles in sensitivity to chemoradiotherapy (CRT) by proving that both peripheral circulating lymphocytes and tumor infiltrating lymphocytes (TILs) strongly correlated with the response rate to CRT and it impacted disease outcome. Aim of the work: We aimed to assess the predictive value of peripheral blood lymphocytes and tumor infiltrating lymphocytes by correlation with regression rate post chemo-radiotherapy in patients with rectal cancer, and to find correlation between peripheral and tissue lymphocytes. Method: Before neoadjuvant, CRT venous blood samples were obtained from 40 patients with rectal cancer, and prior to surgery. Blood cell counts in the samples were analyzed using an automated hematology analyzer and flowcytometry used to analyze lymphocyte subsets. Colonscopic biopsies were obtained before the CRT; the numbers and distributions of $\mathrm{T}$ cells (CD4 \& CD8) were evaluated by immunostaining. Results: Pre CRT peripheral total lymphocytes, T lymphocytes, T helper, T cytotoxic lymphocytes significantly correlated with tumor regression rate $(p=$ $0.04,0.05,0.06,0.04$ respectively). The density of tissue CD4(+) and CD8(+) T cells was highly correlated with tumor regression post CRT ( $p=0.01$ for both). The high expressions of tissue CD4 \& CD 8 were significantly correlated with high number of pretreatment peripheral total lymphocytes, $\mathrm{T}$ lymphocytes, $\mathrm{T}$
\end{abstract}


helper, and $\mathrm{T}$ cytotoxic lymphocytes with significant $p$ value for all. Conclusion: We concluded that peripheral lymphocytic count and its subsets have significant correlation to tissue CD4, CD8 and both can predict pathological response to CRT; enhancement of lymphocytes mediated immune response can help for outcome improvement.

\section{Keywords}

Locally Advanced Rectal Carcinoma, Peripheral Lymphocyte Subsets, Tissue CD4, CD8

\section{Introduction}

Although the last years show much improvement in the plan of management for patients with locally advanced rectal cancer which results in improved outcomes, rectal cancer is still one of the leading causes of cancer related death [1]. Tailoring therapy has become the aim in treatment plan of any cancer patient; guidelines provide standard of care for management of locally advanced rectal cancer (LARC) which is neoadjuvant concurrent modality of Chemo-Radio Therapy (CRT) followed by surgery including: Total Mesorectal Resection (TMR), then adjuvant chemotherapy [2], but we still see variations in individual patient response and outcomes which prove that rectal cancer is still a heterogeneous disease.

Many researches were done to correlate the CRT sensitivity and response with multiple variables [3]. Microenvironment of the tumor and immune response of the host play an essential role in the sensitivity to CRT by proving that both the tumor infiltrating lymphocytes (TILs) density and peripheral blood circulating lymphocytes strongly correlate with the benefit rates to CRT in retrospective studies [4].

The immune system enhances the removal of tumor cells and work for tumor progress control [5]. Previous studies have showed that immunologic response which exists pretreatment may enhance the efficacy of cancer treatment by chemotherapy and radiotherapy modalities [6] [7] [8]. High density of cytotoxic lymphocytes (CD8) in tumor tissue can kill tumor cells when it differentiates into effector cells and this is associated with decreased cancer dissemination and better outcomes [9]. In contrast, increase of infiltrating regulatory T cells (CD4) density, with its suppressor function to other effector $\mathrm{T}$ cell activities, is associated with poorer prognosis in some cancer types [10] [11]. If we detect factors which can predict response to treatment, we can avoid toxic useless modality of treatments and augment modalities for others, individualize our management, and predict oncological disease outcomes [12].

In our study, we aim to assess the predictive value of peripheral lymphocytes, peripheral lymphocytes subsets, and tissue infiltrating lymphocytes (CD4 \& CD8) by correlating to pathological response for post chemoradiotherapy in locally 
advanced rectal cancer and try for the first time to find correlation between peripheral lymphocyte subsets and tissue infiltrating lymphocytes (CD4 \& CD8).

\section{Methods}

\section{Patients and Methods}

This prospective study included 40 patients with new diagnosed Locally Advanced Rectal Cancer (LARC) who were presented to Medical Oncology, Clinical Oncology \& Nuclear Medicine and Surgery departments, Faculty of Medicine, Zagazig University in the period from December 2013 to December 2016. The study protocol was approved by the Ethical Committee of Faculty of Medicine, Zagazig University.

$>$ Clinical data

After baseline workup has been done in the form of colonoscopy and biopsy, pelvic MRI, chest/abdomen/pelvis CT, plus routine renal and liver functions, Carcino Embryonic Antigen (CEA) level, locally advanced rectal cancer had been confirmed.

$>$ Inclusion and exclusion criteria

We included patients with Stage III rectal cancer who confirmed by pelvic MRI to have positive nodal involvement, and/or T3 and T4, adequate blood counts, hepatic, and renal function, the Eastern Cooperative Oncology Group (ECOG) performance status 0 - 2. Patients with distant metastasis, poor performance status were excluded.

- Concomitant Chemo-Radiotherapy

Patients were immobilized in the prone position with a full bladder using a combination of a foam cushion and a prone head cushion. Setup marks were drawn on the patient's skin and the cushion after laser alignment. A planning CT scan was performed using a diagnostic CT scanner. The scan extended from the L2 vertebral body to $2 \mathrm{~cm}$ below the perineum, and axial images were obtained at 5 $\mathrm{mm}$ intervals and imported to the planning system. The $3 \mathrm{D}$ conformal RT was employed in the treatment of all patients involved in the analysis. RT planning was accomplished using a 4-field technique box technique the clinical tumor volume (CTV) included GTV plus a 2-cm margin and nodal drainage. At risk nodes include the presacral, pelvic mesentery, and internal iliac nodes. External iliac nodes included for T4 disease. A boost field included the initial GTV plus $2 \mathrm{~cm}$ and the sacral hollow. All the patients were irradiated on the linear accelerator, with high energy X-rays. Patients received a total dose of 50.4 Gy, Phase 1, 45 Gy in 25 fractions, followed by a Phase 2 boost of $5.4 \mathrm{~Gy}$ in 3 fractions to the primary tumor, with 5 fractions per week (1.8 Gy fraction/day). Radiation therapy was given with concomitant oral 5-Fu chemotherapy capcitabine (oral flurouracil) $825 \mathrm{mg} / \mathrm{m}^{2}$ twice per day during radiation course with or without weekend breaks.

- Surgical procedure

After an average of 6 - 8 weeks of completing CRT, patients underwent surgical resection with negative surgical margin including Total Mesorectal Resec- 
tion (TMR). Surgery were either Lower Anterior Resection (LAR) in 29 patients (72.5\%) or Abdominoperineal Resection (APR) in 11 patients (27.5\%).

$>$ Laboratory methods

Before neoadjuvant CRT, we collected peripheral venous blood samples and 4 - 6 weeks after completion of CRT from our study patients, $2 \mathrm{ml}$ blood were collected in sterile EDTA vacutainer tube; the blood cell counts in the samples were analyzed by automated hematology analyzer (Sysmexxs 1000i manufactured in Japan). Flow cytometery was used to analyze lymphocyte subsets. The surface staining was done by adding $10 \mu \mathrm{l}$ of each mAbs to $100 \mu \mathrm{l}$ of anticoagulated blood in the same tube, incubation of tube was done in the dark, for $30 \mathrm{~min}$ at $4{ }^{\circ} \mathrm{C}$, we did washing twice with FACS washing buffer. Lysing reagent was added to each tube, inverted once, kept for 3 minutes. Finally, $0.5 \mathrm{ml}$ of Phosphate buffer saline (PBS) was added on the washed cells. Gating for the region of lymphocytes, and flow-cytometric analysis for each cell phenotype on 10,000 events was detected on the FACSCalibur flow cytometer (Becton Dickinson) using he Multiset software package (Becton Dickinson), and the CellQuest software was used for data analysis. We used a combination of isothiocyanate (FITC) and phycoerythrin (PE)-conjugated monoclonal antibody (Becton Dickinson, San Jose, CA, USA) to identify lymphocyte subsets, as follows: T lymphocyte: CD3(+)/CD19(-), B lymphocytes: CD3(-)/CD19(+), helper T lymphocytes (Th lymphocytes), CD3 $(+) / C D 4(+)$, cytotoxic T lymphocytes (Tc lymphocytes) CD3(+)/CD8(+) and for the natural killer cells: CD3(-)/CD56(+) as shown in Figure 1 [13].

$>$ Routine Histopathological method

Colonscopic biopsy samples which obtained from the 40 patients $\mathrm{d}$ before the start of CRT, fixed in $10 \%$ formalin solution, then put in paraffin, serial-step sections of the blocks were cut with $3 \mu \mathrm{m}$ thickness, stained by routine hematoxylin $\&$ eosin stains and diagnosed in Pathology Department. Pathologic staging according to the seventh edition of the American Joint Committee on Cancer staging system (AJCC-7) classification was used [14]; and for grading we depend on the World Health Organization (WHO) classification [15].

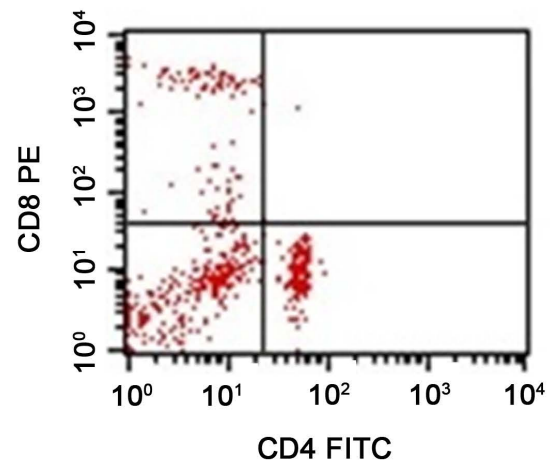

(a)

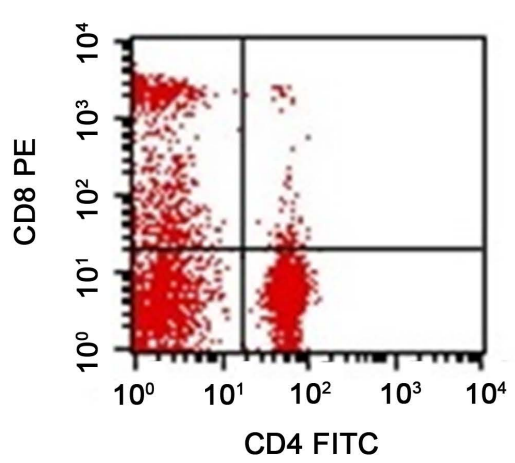

(b)

Figure 1. Show dot blot analysis of flow cytometry data of CD4/CD8 of two cases revealed. (a) A case with T-helper (16\%) (768 cell/ $\mu \mathrm{l})$ and T-cytotoxic (10\%) (480 cell/ $\mu \mathrm{l})$ with low absolute count; (b) A case with T-helper (42.9\%) (4804.8 cell/ $\mu \mathrm{l})$ and T-cytotoxic (23.6\%) (2643.2 cell/ $\mu$ l) with high absolute count. 
Immunohistochemical method:

The $\mathrm{T}$ cells numbers and distribution in biopsy samples before CRT were assessed with immunohistochemical staining with Abs against CD4 and CD8, it was performed by streptavidin biotin technique as described by Hsu and Shinto [16] [17]. We incubated sections with primary rabbit polyclonal anti-CD8 antibody ab85792 and mouse monoclonal [mAb51312] to CD4 (1:100 dilutions (Abcam, Cambridge, MA, USA)).We used sections of human tonsils as positive controls for both markers. For negative controls, primary antibodies were omitted and replaced with normal saline. Under light microscope, at magnification of $400 \times$ in three different sections, the number of immunoreactive lymphocytes was counted in a randomly selected field CD4 \& CD8 expressions scoring.

$>\mathrm{CD} 4$ \& CD8 expressions scoring

In the densest field of CD8 \& CD4 positively stained cells within epithelial compartments we counted the intraepithelial lymphocytes (IELs). TIL scoring of tumors was performed semiquantitatively by measuring the densities of CD8, CD4 cells as described by Dahlin [18], 1) no, or sporadic cells; 2) moderate numbers of cells; 3) abundant occurrence of cells; and 4) highly abundant occurrence of cells]. TILs were evaluated in the following three different areas of the tumor: the intraepithelial compartment (cells within tumor cell nests); the stroma (cells within the intratumoural stroma) and the tumour periphery (cells localized in tumour periphery). Three random fields were examined, whereas necrotic areas were excluded from the measurements. The sum of the individual scores from the three tumor areas (intra-epithelial compartment, stroma and tumour periphery) is the measurement of the total score for CD8, CD4, respectively. The total score ranged from 3 to 12 , to separate the patient cohort into two groups with either low or high CD8, CD4 expressions we used the median value as a cutoff point.

Pathological response evaluation

Pathological examination of surgical tissue postoperative to define who achieved complete remission, pathological $\mathrm{N}$ and $\mathrm{T}$ (ypCR was defined as the absence of any tumor cells in the operative pathology specimen defined by ypT0pN0). Regression grade evaluated depending on AJJC grading system which define 4 regression grades (G0 no residual tumor cells detected, G1 single cell or small group of cells, G2 residual cancer with desmoplastic response and G3 minimal evidence of tumor response).

\section{Statistical Analysis}

SPSS 22.0 for windows (SPSS Inc., Chicago, IL, USA), MedCalc windows (MedCalc Software bvba 13, Ostend, Belgium) and Microsoft Office Excel 2010 for windows (Microsoft Cor., Redmond, WA, USA) were used. Mann Whitney U test was used for non-normally distributed variables. Percent of categorical variables were compared using Pearson's Chi-square test or Fisher's exact test when was appropriate. Receiver operating characteristic (ROC) curve analysis was used to identify optimal cut-off values of leukocytes subpopulations ratio with maximum sensitivity and specificity for prediction of high pathological regression rate fol- 
lowing CRT. Univariate logistic regression analysis was done for clino-pathological parameters to find independent predictors of high pathological regression rate following CRT, any variables had $p$-value $<0.20$ was entered in backward multivariate logistic regression model.

\section{Ethical Considerations}

Informed consent was obtained from all participants included in the study. All procedures performed in studies involving human participants were in accordance with the ethical standards of the institutional and/or national research committee and with the 1964 Helsinki declaration and its later amendments or comparable ethical standards.

\section{Results}

\subsection{Patient Characteristics}

Our study included 40 patients with confirmed locally advanced rectal cancer, 26 (65\%) of them were males and 14 (35\%) were females with age range from 33 70 median; 59.5 years. $33(82.5 \%)$ cases were conventional adenocarcinoma and $7(17.5 \%)$ cases were mucoid carcinoma. Pretreatment positive $\mathrm{cN}$ were seen in $75 \%$ of our patients (all details are presented in Table 1).

\subsection{Clinical Results}

There was a significant correlation between pretreatment clinical T \& $\mathrm{N}$ and post treatment pathological $\mathrm{T}$, pathological $\mathrm{N}$ ( $p$-value 0.004 and 0.015 respectively). We did not found any significant correlation between any one of clinicopathological parameters and peripheral lymphocyte counts either pre or post CRT. Postsurgery, our patients divided into 2 groups depending on AJJC regression grade; high responders G0, 1 (Hi-R) achieved in 18 patients including 6 patients with CpCR (15\%), and low responders G2, 3 (Lo-R) seen in 22 patients (55\%). By using univariate analysis we found pretreatment age and clinical $\mathrm{T}$ were associated with good response (Hi-R) significantly ( $p$-value 0.009 and 0.003 respectively) Table 2.

For pre chemoradiotherapy counts of TLC, ANC, peripheral lymphocyte count and counts of lymphocytes subsets (T lymphocytes, Th, Tc) all showed higher median values in patients with high regression rate (Hi-R) compared to low regression (Lo-R) group as shown in Figure 2(a). Figure 2(b) also showed the same trend when comparing high regression to low regression group; median values for post chemo radiotherapy lab all were higher in high responders (Table 3).

We found significant positive correlation between higher TLC, lymphocyte count, $\mathrm{T}$ lymphocytes, $\mathrm{Th}, \mathrm{Tc}$ and regression rate for pre-CRT values, but for post CRT results we did not found any significant correlation with any of lymphocytes, or lymphocytes subsest Table 4 .

\subsection{Immunohistochemical Results}

CD4 expression: High expression of CD4 was detected in 19 (47.5\%) patients 
Table 1. Clinicopathological characteristics of the rectal carcinoma patients $(\mathrm{N}=40)$.

\begin{tabular}{|c|c|c|}
\hline \multirow{2}{*}{ Characteristics } & \multicolumn{2}{|c|}{ All $(\mathrm{N}=40)$} \\
\hline & No. & (\%) \\
\hline Age (years) & $59.50(33-70)$ & \\
\hline$\leq 60$ years & 24 & $(60 \%)$ \\
\hline$>60$ years & 16 & $(40 \%)$ \\
\hline \multicolumn{3}{|l|}{ Sex } \\
\hline Male & 26 & $(65 \%)$ \\
\hline Female & 14 & $(35 \%)$ \\
\hline \multicolumn{3}{|l|}{ Pattern of gross } \\
\hline Fungating & 26 & $(65 \%)$ \\
\hline Ulcerating & 9 & $(22.5 \%)$ \\
\hline Annular & 5 & $(12.5 \%)$ \\
\hline \multicolumn{3}{|l|}{ Histopathological subtypes } \\
\hline Conventional adenocarcinoma & 33 & $(82.5 \%)$ \\
\hline Mucoid carcinoma & 7 & $(17.5 \%)$ \\
\hline \multicolumn{3}{|l|}{ Grade } \\
\hline Poorly differentiated & 9 & $(22.5 \%)$ \\
\hline Moderately differentiated & 12 & $(30 \%)$ \\
\hline Well differentiated & 19 & $(47.5 \%)$ \\
\hline Distance from anal verge $(\mathrm{cm})$ & $6(2-12)$ & \\
\hline$<5 \mathrm{~cm}$ & 14 & $(35 \%)$ \\
\hline $5-10 \mathrm{~cm}$ & 18 & $(45 \%)$ \\
\hline$>10 \mathrm{~cm}$ & 8 & $(20 \%)$ \\
\hline \multicolumn{3}{|l|}{ Clinical T } \\
\hline $\mathrm{T} 1$ & $2(5 \%)$ & \\
\hline $\mathrm{T} 2$ & $5(12.5 \%)$ & \\
\hline T3 & $24(60 \%)$ & \\
\hline $\mathrm{T} 4$ & $9(22.5 \%)$ & \\
\hline \multicolumn{3}{|l|}{ Clinical N } \\
\hline No & $10(25 \%)$ & \\
\hline N1 & $14(35 \%)$ & \\
\hline $\mathrm{N} 2$ & $12(30 \%)$ & \\
\hline N3 & $4(10 \%)$ & \\
\hline \multicolumn{3}{|l|}{ Lymphatic invasion } \\
\hline Absent & 29 & $(72.5 \%)$ \\
\hline Present & 11 & $(27.5 \%)$ \\
\hline \multicolumn{3}{|l|}{ Venous invasion } \\
\hline Absent & 24 & $(60 \%)$ \\
\hline Present & 16 & $(40 \%)$ \\
\hline \multicolumn{3}{|l|}{ pCR } \\
\hline No & 34 & $(85 \%)$ \\
\hline Yes & 6 & $(15 \%)$ \\
\hline \multicolumn{3}{|l|}{ Regression group } \\
\hline 3 & 8 & $(20 \%)$ \\
\hline 2 & 14 & $(35 \%)$ \\
\hline 1 & 12 & $(30 \%)$ \\
\hline 0 & 6 & $(15 \%)$ \\
\hline \multicolumn{3}{|l|}{ Regression rate } \\
\hline Lo-R & 22 & $(55 \%)$ \\
\hline $\mathrm{Hi}-\mathrm{R}$ & 18 & $(45 \%)$ \\
\hline
\end{tabular}

Categorical variables were expressed as number (percentage). Continuous variables were expressed as median (range). pCR (pathological Complete Remission), Lo-R (Low Regression), Hi-R (High Regression). 
Table 2. Univariate and multivariate analysis of clinical factors in predicting CRT response in rectal carcinoma patients $(\mathrm{N}=40)$.

\begin{tabular}{cccccc}
\hline & & Univariate & \multicolumn{2}{c}{ Multivariate } & \\
\cline { 3 - 6 } & & $p$-value & OR $(95 \% \mathrm{CI})$ & $p$-value \\
\hline Gender & Male vs. Female & 0.64 & & & \\
Age & $\leq 60$ years vs. $>60$ years & 0.04 & 11.936 & $(1.241-114.760)$ & 0.03 \\
Grade & Moderate vs. poor & 0.27 & & & \\
Distance from anal verge & $>10$ vs. $\leq 10 \mathrm{~cm}$ & 0.75 & & & 0.09 \\
Clinical T & cT1-2 vs. cT3-4 & 0.04 & 11.967 & $(0.666-214.896)$ & \\
Clinical N & cN0 vs. cN + & 0.08 & & & \\
Pre-CRT TLC & $>5200$ vs. $\leq 5200$ cell/ $/ \mu \mathrm{L}$ & 0.09 & & & \\
Pre-CRT Lymphocytes & $>1850$ vs. $\leq 1850$ cell/ $/ \mu \mathrm{L}$ & 0.04 & 29.854 & $(1.696-525.525)$ & 0.02 \\
Pre-CRT T cells & $>1310$ vs. $\leq 1310$ cell/ $/ \mu \mathrm{L}$ & 0.09 & & & \\
Pre-CRT T helper & $>971$ vs. $\leq 971$ cell/ $/ \mu \mathrm{L}$ & 0.04 & & & \\
Pre-CRT T cytotoxic & $>460$ vs. $\leq 460$ cell/ $/ \mu \mathrm{L}$ & 0.04 & 10.861 & $(0.763-154.628)$ & 0.08 \\
\hline
\end{tabular}

OR: Odds Ratio; $95 \%$ CI: 95\% confidence interval; $p<0.05$ is significant. CRT (Chemoradiotherapy), TLC (Total Leucocyte Count).

Table 3. Leukocytes subpopulation as predictors for high pathological regression of rectal adenocarcinoma after preoperative CRT: ROC curve.

\begin{tabular}{|c|c|c|c|c|c|c|c|}
\hline $\begin{array}{l}\text { Cut-off } \\
\text { values }\end{array}$ & $\begin{array}{c}\text { SN \% } \\
(95 \% \mathrm{CI})\end{array}$ & $\begin{array}{c}\mathrm{SP} \% \\
(95 \% \mathrm{CI})\end{array}$ & $\begin{array}{c}\text { PPV \% } \\
(95 \% \text { CI) }\end{array}$ & $\begin{array}{c}\text { NPV \% } \\
(95 \% \mathrm{CI})\end{array}$ & $\begin{array}{l}\text { Accuracy } \\
(95 \% \text { CI })\end{array}$ & $\begin{array}{l}\text { AUROC } \\
(95 \% \mathrm{CI})\end{array}$ & $p$-value \\
\hline \multicolumn{8}{|l|}{ Pre-CRT } \\
\hline TLC & $88.9 \%$ & $54.6 \%$ & $61.5 \%$ & $85.7 \%$ & $70 \%$ & 0.692 & \multirow{2}{*}{0.03} \\
\hline \multicolumn{7}{|c|}{$>5200 \mathrm{cell} / \mu \mathrm{L}(65.3-98.6)(32.2-75.6)(40.1-80.1)(57.2-98.2) \quad(47.1-86) \quad(0.526-0.828)$} & \\
\hline Lymphocytes & $94.4 \%$ & $59.1 \%$ & $65.4 \%$ & $92.9 \%$ & $75 \%$ & 0.749 & \multirow{2}{*}{0.02} \\
\hline \multicolumn{7}{|c|}{$>1850$ cell $/ \mu \mathrm{L}(72.7-99.9)(36.4-79.3)(44.3-82.8)(66.1-99.8)(52.7-88.6)(0.587-0.872)$} & \\
\hline T cells & $88.9 \%$ & $54.6 \%$ & $61.5 \%$ & $85.7 \%$ & $70 \%$ & 0.764 & \multirow{2}{*}{$<0.01$} \\
\hline \multicolumn{5}{|c|}{$>1310$ cell $/ \mu \mathrm{L}(65.3-98.6)(32.2-75.6)(40.1-80.1)(57.2-98.2)$} & $(47.1-86)$ & $(0.603-0.883)$ & \\
\hline T helper & $94.4 \%$ & $59.1 \%$ & $65.4 \%$ & $92.9 \%$ & $75 \%$ & 0.758 & \multirow{2}{*}{$<0.01$} \\
\hline \multicolumn{7}{|c|}{$>971 \mathrm{cell} / \mu \mathrm{L}(72.7-99.9)(36.4-79.3)(44.3-82.8)(66.1-99.8)(52.7-88.6)(0.596-0.879)$} & \\
\hline T cytotoxic & $55.6 \%$ & $95.5 \%$ & $90.9 \%$ & $72.4 \%$ & $77.5 \%$ & 0.750 & \multirow{2}{*}{0.02} \\
\hline \multicolumn{7}{|l|}{$>460 \mathrm{cell} / \mu \mathrm{L}$} & \\
\hline \multicolumn{8}{|l|}{ Post-CRT } \\
\hline TLC & $77.8 \%$ & $77.3 \%$ & $73.7 \%$ & $81 \%$ & $77.5 \%$ & 0.745 & \multirow{2}{*}{0.02} \\
\hline \multicolumn{7}{|c|}{$>5300 \mathrm{cell} / \mu \mathrm{L}(52.4-93.6)(54.6-92.2)(48.8-90.9)(57.4-94.8)(53.6-92.8)(0.583-0.869)$} & \\
\hline $\mathrm{ANC}$ & $77.8 \%$ & $68.2 \%$ & $66.7 \%$ & $78.9 \%$ & $72.5 \%$ & 0.702 & \multirow{2}{*}{0.02} \\
\hline \multicolumn{4}{|c|}{$>2500$ cell $/ \mu \mathrm{L}(52.4-93.6)(45.1-86.1)(43-85.4)$} & $(53.6-94.2)$ & $(48.4-89.5)$ & $(0.537-0.836)$ & \\
\hline Lymphocytes & $88.9 \%$ & $54.6 \%$ & $61.5 \%$ & $85.7 \%$ & $70 \%$ & 0.703 & \multirow{2}{*}{0.02} \\
\hline$\leq 36.2 \%$ & $(65.3-98.6$ & $(32.2-75.6)$ & $(40.1-80.1)$ & $(57.2-98.2)$ & $(47.1-86)$ & $(0.538-0.837)$ & \\
\hline NLR & $77.8 \%$ & $68.2 \%$ & $66.7 \%$ & $78.9 \%$ & $72.5 \%$ & 0.756 & \multirow{2}{*}{0.01} \\
\hline$>1.47$ & (52.4 - 93.6 & $(45.1-86.1)$ & $(43-85.4)$ & $(53.6-94.2)$ & $(48.4-89.5)$ & $(0.595-0.878)$ & \\
\hline
\end{tabular}

ROC curve: Receiver Operating Characteristic curve; SN: Sensitivity; SP: Specificity; PPV: Positive Predictive Value; NPV: Negative Predictive Value; AUROC: Area under Receiver Operating Characteristic Curve; 95\% CI: 95\% Confidence Interval; $p<0.05$ is significant. CRT (Chemoradiotherapy), TLC (Total Leucocyte Count), ANC (Absolute Neutrophils Count), NK (Natural Killer), NLR (Neutrophils Lymphocytes Ratio). 
Table 4. Relation between pathological response and laboratory findings of the rectal carcinoma patients $(\mathrm{N}=40)$.

\begin{tabular}{|c|c|c|c|c|c|c|c|}
\hline \multirow{3}{*}{ Variables } & \multirow{2}{*}{\multicolumn{2}{|c|}{$\begin{array}{c}\text { All } \\
(\mathrm{N}=40)\end{array}$}} & \multicolumn{4}{|c|}{ Regression rate } & \multirow{3}{*}{$p$-value } \\
\hline & & & \multicolumn{2}{|r|}{$\begin{array}{c}\text { Lo-R } \\
(\mathrm{N}=22)\end{array}$} & \multicolumn{2}{|c|}{$\begin{array}{c}\mathrm{Hi}-\mathrm{R} \\
(\mathrm{N}=18)\end{array}$} & \\
\hline & Median & (Range) & Median & (Range) & Median & (Range) & \\
\hline \multicolumn{8}{|l|}{ Pre-CRT } \\
\hline TLC $($ cell $/ \mu \mathrm{L})$ & 7100 & $(4000-14,100)$ & 5100 & $(4000-13,000)$ & 8250 & $\begin{array}{l}(4200- \\
14,100)\end{array}$ & $0.04 \bullet$ \\
\hline ANC $($ cell/ $\mu \mathrm{L})$ & 3950 & $(2000-9120)$ & 3050 & $(2000-9120)$ & 4529.50 & $(2200-9120)$ & $0.07 \bullet$ \\
\hline ANC (\%) & 56.60 & $(38-70.20)$ & 57.60 & $(45.50-70.20)$ & 55.35 & $(38-64.70)$ & $0.18^{*}$ \\
\hline $\begin{array}{l}\text { Lymphocyte } \\
(\text { cell } / \mu \mathrm{L})\end{array}$ & 2357 & $(980-4215)$ & 1820 & $(980-3450)$ & 2920 & $(1150-4215)$ & $0.04^{\star}$ \\
\hline Lymphocyte (\%) & 33.40 & $(22.90-43.60)$ & 32.90 & $(22.90-40.50)$ & 33.40 & $(25.90-43.60)$ & $0.12^{*}$ \\
\hline $\mathrm{T}$ cells $($ cell $/ \mu \mathrm{L})$ & 1532 & $(686-3315)$ & 1302.50 & $(686-2240)$ & 2044 & $(805-3315)$ & $0.05^{*}$ \\
\hline $\begin{array}{l}\text { T helper } \\
\text { (cell/ } / \mu \mathrm{L})\end{array}$ & 1158 & $(515-2453)$ & 910 & $(515-1840)$ & 1547 & $(604-2453)$ & $0.06 \bullet$ \\
\hline $\begin{array}{l}\text { T cytotoxic } \\
(\text { cell } / \mu \mathrm{L})\end{array}$ & 350 & $(102-862)$ & 283.50 & $(102-660)$ & 480 & $(120-862)$ & $0.04^{*}$ \\
\hline $\begin{array}{l}\text { NK cells } \\
(\text { cell/ } \mu \mathrm{L})\end{array}$ & 152.50 & $(68-423)$ & 137 & $(68-400)$ & 167.50 & $(80-423)$ & $0.18 \bullet$ \\
\hline $\mathrm{B}$ cells $(\mathrm{cell} / \mu \mathrm{L})$ & 562.50 & $(294-970)$ & 485 & $(294-960)$ & 602.50 & $(345-970)$ & $0.05^{*}$ \\
\hline NLR & 1.79 & $(1.20-3.10)$ & 1.72 & $(1.30-3.10)$ & 1.66 & $(1.20-2.29)$ & $0.19^{*}$ \\
\hline CEA $(\mu \mathrm{g} / \mathrm{L})$ & 57.50 & $(3-390)$ & 65 & $(3-390)$ & 49.50 & $(5-360)$ & $0.97 \bullet$ \\
\hline \multicolumn{8}{|l|}{ Post-CRT } \\
\hline TLC (cell/ $\mu \mathrm{L})$ & 5200 & $(3000-8700)$ & 4250 & $(3000-8180)$ & 6120.50 & $(3200-8700)$ & $0.08 \bullet$ \\
\hline ANC $($ cell $/ \mu \mathrm{L})$ & 2600 & $(1500-5120)$ & 2275 & $(1500-4350)$ & 3275 & $(1500-5120)$ & 0.03 \\
\hline ANC (\%) & 52.65 & $(38.40-66.40)$ & 51.30 & $(42.50-64.50)$ & 55.50 & $(38.40-66.40)$ & $0.99^{*}$ \\
\hline $\begin{array}{l}\text { Lymphocyte } \\
(\text { cell/ } / \mu \mathrm{L})\end{array}$ & 1900 & $(990$ - 3015) & 1615 & $(990$ - 3015) & 2007 & $(1100-2710)$ & $0.18^{*}$ \\
\hline Lymphocyte (\%) & 34.10 & $(21.10-46.40)$ & 36.95 & $(25.60-46.40)$ & 33.20 & $(21.10-40.60)$ & $0.06^{*}$ \\
\hline $\begin{array}{l}\mathrm{T} \text { cells } \\
\text { (cell/ } / \mu \mathrm{L})\end{array}$ & 1205 & $(590-2440)$ & 1132.50 & $(740-2000)$ & 1410.50 & $(590-2440)$ & $0.15 \bullet$ \\
\hline $\begin{array}{l}\mathrm{T} \text { helper } \\
(\text { cell } / \mu \mathrm{L})\end{array}$ & 900 & $(410-1820)$ & 830 & $(585-1500)$ & 950 & $(410-1820)$ & $0.25 \bullet$ \\
\hline $\begin{array}{l}\text { T cytotoxic } \\
(\text { cell/ } / \mu \mathrm{L})\end{array}$ & 367.50 & $(130-760)$ & 277.50 & $(130-710)$ & 450 & $(180-760)$ & $0.07 \bullet$ \\
\hline $\begin{array}{l}\text { NK cells } \\
\text { (cell/ } \mu \mathrm{L})\end{array}$ & 141 & $(45-357)$ & 131.50 & $(80-320)$ & 160 & $(45-357)$ & $0.22 \bullet$ \\
\hline $\mathrm{B}$ cells $($ cell $/ \mu \mathrm{L})$ & 380 & $(125-780)$ & 348 & $(125-780)$ & 420 & $(150-630)$ & $0.76^{*}$ \\
\hline NLR & 1.50 & $(1.01-2.52)$ & 1.35 & $(1.01-2.52)$ & 1.75 & $(1.23-2.12)$ & $0.06 \bullet$ \\
\hline CEA $(\mu \mathrm{g} / \mathrm{L})$ & 10 & $(2-60)$ & 18 & $(2-55)$ & 7.50 & $(2-60)$ & $0.42 \bullet$ \\
\hline
\end{tabular}

Continuous variables were expressed as median (range); ${ }^{\star}$ Independent samples Student's t-test; •Mann Whitney U test; $p<0.05$ is significant. TLC (Total Leucocyte Count), ANC (Absolute Neutrophils Count), NK (Natural Killer), NLR (Neutrophils Lymphocytes Ratio), CEA (Carcinoembyonic Antigen). 


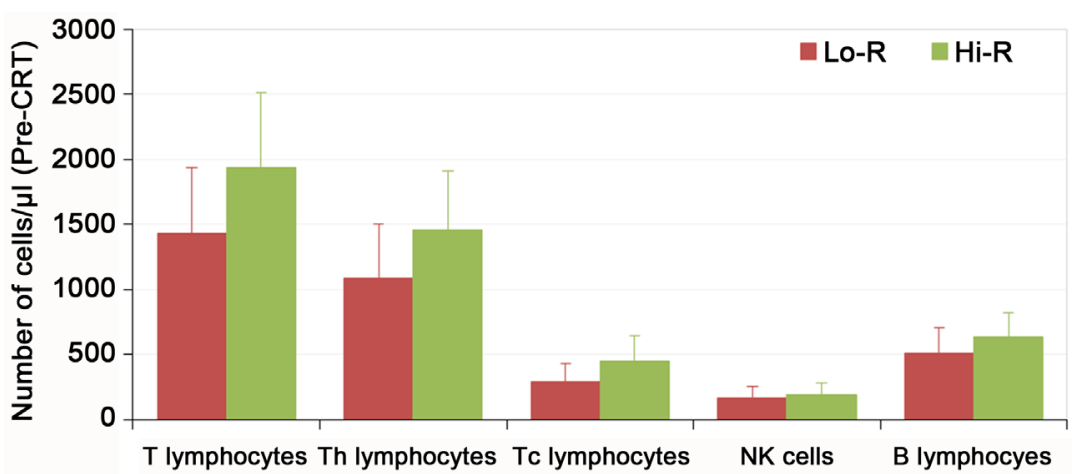

(a)

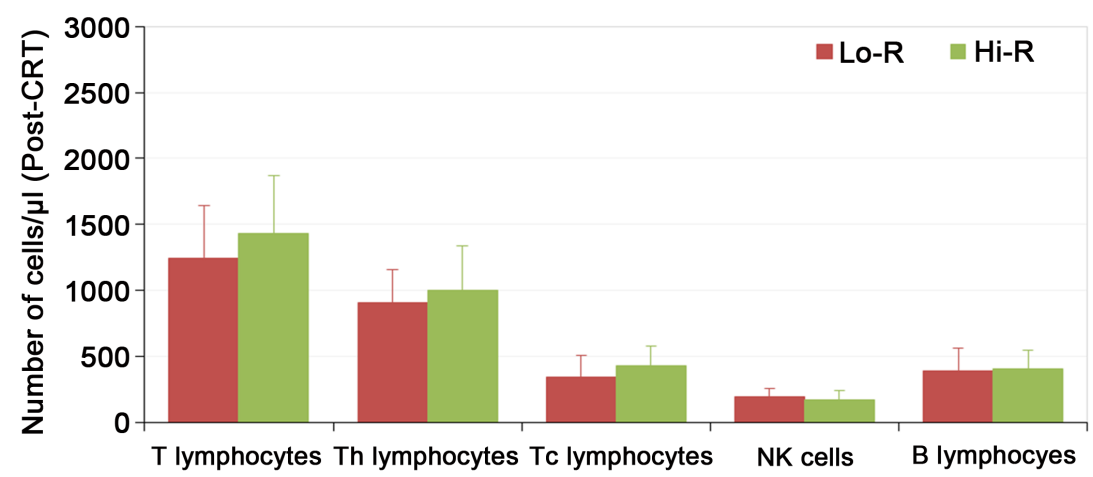

(b)

Figure 2. The correlation between the pathological response and number of peripheral blood lymphocyte subsets; (a) Pre-CRT; (b) Post-CRT. Data are expressed as mean + $1 \mathrm{SD}$.

(Figure 3(a)). No significant correlation was found between CD4 expression and age, sex of our patients, histopathological subtype, gross pattern or grade of the carcinoma. The high expressions of CD4 in rectal carcinoma was significantly correlated to cT stage $(p=0.01)$, high number of lymphocytes $(p=0.05)$, T helper $(p=0.01)$ and Tc lymphocytes $(p=0.01)$ circulating in peripheral blood (Table 5). CD8 expression: high expression of CD8 was detected in 22 patients (55\%) (Figure $3(c)$ ) the high expressions of CD8 was significantly correlated with age of the patient $(p=0.01)$, cT stage $(p=0.01)$, high peripheral lymphocyte count $(p=$ $0.27)$, high $\mathrm{T}$ cells $(p=0.03)$, high $\mathrm{T}$ cytotoxic lymphocytes circulating in peripheral blood $(p=0.010)$ (Table 5), but no significant correlation was found between its expression and sex of our patients, histopathological subtype, gross pattern or grade of the carcinoma. Expressions of both (CD 4 \& CD8) were significantly positively correlated with each other $(p<0.01)$. When the numbers were counted in each case, the densities of $\mathrm{CD} 4(+)$ and $\mathrm{CD} 8(+) \mathrm{T}$ cells showed a strong association more importantly; the density of $\mathrm{CD} 4(+)$ as well as $\mathrm{CD} 8(+) \mathrm{T}$ cells was highly correlated with tumor response to CRT. All who achieve Cp CR (6 patients) were with high tissue CD4 ( $p$-value 0.07$) \& \operatorname{CD} 8$ ( $p$-value 0.02 ) in contrast to those with low CD4 \& CD8 densities as no one of them achieved CR. High CD4 \& CD8 expressions showed statistically highly significant correlations with high regression rate $(p<0.01)$ (Table 6). 
Table 5. Relation between peripheral lymphocytes and CD4 \& CD8 IHC staining in 40 rectal carcinoma patients.

\begin{tabular}{|c|c|c|c|c|c|c|c|}
\hline \multirow{3}{*}{ Characteristics } & \multirow{2}{*}{\multicolumn{2}{|c|}{$\begin{array}{c}\text { All } \\
(\mathrm{N}=40)\end{array}$}} & \multicolumn{4}{|c|}{$\mathrm{CD} 4$} & \multirow{3}{*}{$p$-value } \\
\hline & & & \multicolumn{2}{|r|}{$\begin{array}{c}\text { Low } \\
(\mathrm{N}=21)\end{array}$} & \multicolumn{2}{|r|}{$\begin{array}{c}\text { High } \\
(\mathrm{N}=19)\end{array}$} & \\
\hline & No. & (\%) & No. & $(\%)$ & No. & (\%) & \\
\hline \multicolumn{8}{|l|}{$\begin{array}{c}\text { Pre-CRT } \\
\text { laboratory findings }\end{array}$} \\
\hline TLC (cell/ $\mu \mathrm{L})$ & 7100 & $(4000-14,100)$ & 5200 & $(4000-13,000)$ & 8100 & $(4200-14,100)$ & $0.07 \bullet$ \\
\hline ANC (cell/ $\mu \mathrm{L})$ & 3950 & $(2000-9120)$ & 3100 & $(2000-9120)$ & 4000 & $(2200-9120)$ & $0.19 \bullet$ \\
\hline ANC (\%) & 56.60 & $(38-70.20)$ & 57.70 & $(38-70.20)$ & 55.70 & $(45.50-64.70)$ & $0.51^{\star}$ \\
\hline Lymphocyte (cell/ $\mu \mathrm{L})$ & 2357 & $(980-4215)$ & 1840 & $(980-3450)$ & 2940 & $(1150-4215)$ & $0.05^{*}$ \\
\hline Lymphocyte (\%) & 33.40 & $(22.90-43.60)$ & 32.10 & $(22.90-38.40)$ & 33.90 & $(25.90-43.60)$ & $0.04^{*}$ \\
\hline $\mathrm{T}$ cells $($ cell $/ \mu \mathrm{L})$ & 1532 & $(686-3315)$ & 1310 & $(686-2240)$ & 2058 & $(805-3315)$ & $0.05^{*}$ \\
\hline $\mathrm{T}$ helper (cell/ $\mu \mathrm{L})$ & 1158 & $(515-2453)$ & 920 & $(515-1840)$ & 1544 & $(604-2453)$ & $0.01^{*}$ \\
\hline $\mathrm{T}$ cytotoxic $($ cell $/ \mu \mathrm{L})$ & 350 & $(102-862)$ & 289 & $(102-460)$ & 490 & $(120-862)$ & $0.01^{*}$ \\
\hline $\mathrm{NK}$ cells (cell/ $\mu \mathrm{L})$ & 152.50 & $(68-423)$ & 145 & $(68-400)$ & 155 & $(80-423)$ & $0.28 \bullet$ \\
\hline B cells $($ cell $/ \mu \mathrm{L})$ & 562.50 & $(294-970)$ & 490 & $(294-960)$ & 605 & $(345-970)$ & $0.10^{*}$ \\
\hline \multirow{2}{*}{ Characteristics } & & $\begin{array}{c}\text { All } \\
(\mathrm{N}=40)\end{array}$ & \multicolumn{4}{|c|}{$\mathrm{CD} 8$} & value \\
\hline & No. & (\%) & No. & (\%) & No. & (\%) & \\
\hline $\begin{array}{c}\text { Pre-CRT } \\
\text { laboratory findings }\end{array}$ & & & & & & & \\
\hline TLC $($ cell $/ \mu \mathrm{L})$ & 7100 & $(4000-14,100)$ & 6050 & $(4000-13,000)$ & 7700 & $(4200-14,100)$ & 0.25 \\
\hline ANC $($ cell $/ \mu \mathrm{L})$ & 3950 & $(2000-9120)$ & 3300 & $(2000-9120)$ & 4000 & $(2100-9120)$ & $0.44 \bullet$ \\
\hline $\operatorname{ANC}(\%)$ & 56.60 & $(38-70.20)$ & 58.45 & $(45.50-70.20)$ & 55.35 & $(38-64.70)$ & $0.08^{*}$ \\
\hline Lymphocyte (cell/ $/ \mu \mathrm{L})$ & 2357 & $(980-4215)$ & 1820 & $(980-3450)$ & 2900 & $(1150-4215)$ & $0.03^{*}$ \\
\hline Lymphocyte (\%) & 33.40 & $(22.90-43.60)$ & 30.60 & $(22.90-38.40)$ & 34.55 & $(25.90-43.60)$ & $0.01^{\star}$ \\
\hline $\mathrm{T}$ cells $($ cell $/ \mu \mathrm{L})$ & 1532 & $(686-3315)$ & 1330 & $(686-2240)$ & 2025 & $(805-3315)$ & $0.03^{*}$ \\
\hline $\mathrm{T}$ helper (cell/ $/ \mu \mathrm{L})$ & 1158 & $(515-2453)$ & 910 & $(515-1840)$ & 1495 & $(604-2453)$ & $0.05^{\star}$ \\
\hline $\mathrm{T}$ cytotoxic $($ cell $/ \mu \mathrm{L})$ & 350 & $(102-862)$ & 299.50 & $(102-460)$ & 417 & $(120-862)$ & $0.01^{*}$ \\
\hline NK cells (cell/ $\mu \mathrm{L})$ & 152.50 & $(68-423)$ & 152.50 & $(68-400)$ & 152.50 & $(80-423)$ & $0.43 \bullet$ \\
\hline B cells $($ cell $/ \mu \mathrm{L})$ & 562.50 & $(294-970)$ & 446.50 & $(294-960)$ & 600 & $(345-970)$ & $0.09 \bullet$ \\
\hline
\end{tabular}

Categorical variables were expressed as number (percentage). Continuous variables were expressed as median (range). ${ }^{\star}$ Independent samples Student’s t-test; •Mann Whitney U test; $¥$ Chi-square test; §Chi-square test for trend; $p<0.05$ is significant.

Table 6. Effect of CD4 \& CD8 IHC staining on pathological response to CRT and postoperative histopathological examination of the rectal carcinoma patients $(\mathrm{N}=40)$.

\begin{tabular}{|c|c|c|c|c|c|c|c|}
\hline \multirow{3}{*}{ Characteristics } & \multirow{2}{*}{\multicolumn{2}{|c|}{$\begin{array}{c}\text { All } \\
(\mathrm{N}=40)\end{array}$}} & \multicolumn{4}{|c|}{ CD4 } & \multirow{3}{*}{$p$-value } \\
\hline & & & \multicolumn{2}{|c|}{ Low $(\mathrm{N}=21)$} & \multicolumn{2}{|c|}{$\operatorname{High}(\mathrm{N}=19)$} & \\
\hline & No. & $(\%)$ & No. & $(\%)$ & No. & $(\%)$ & \\
\hline \multicolumn{8}{|l|}{ yp T } \\
\hline урТ0 & 6 & $(15 \%)$ & 0 & $(0 \%)$ & 6 & $(31.6 \%)$ & $0.01 \S$ \\
\hline ypT1 & 8 & $(20 \%)$ & 2 & $(9.5 \%)$ & 6 & $(31.6 \%)$ & \\
\hline урТ2 & 15 & $(37.5 \%)$ & 9 & $(42.9 \%)$ & 6 & $(31.6 \%)$ & \\
\hline урТ3 & 11 & $(27.5 \%)$ & 10 & $(47.6 \%)$ & 1 & $(5.3 \%)$ & \\
\hline ypN & & & & & & & \\
\hline
\end{tabular}




\section{Continued}

\begin{tabular}{|c|c|c|c|c|c|c|c|}
\hline ypN0 & 21 & $(52.5 \%)$ & 9 & $(42.9 \%)$ & 12 & $(63.2 \%)$ & \multirow{3}{*}{$0.19 \$$} \\
\hline ypN1 & 18 & $(45 \%)$ & 12 & $(57.1 \%)$ & 6 & $(31.6 \%)$ & \\
\hline ypN2 & 1 & $(2.5 \%)$ & 0 & $(0 \%)$ & 1 & $(5.3 \%)$ & \\
\hline \multicolumn{8}{|l|}{ Lymphatic invasion } \\
\hline Absent & 29 & $(72.5 \%)$ & 17 & $(81 \%)$ & 12 & $(63.2 \%)$ & \multirow{2}{*}{$0.21 \S$} \\
\hline Present & 11 & $(27.5 \%)$ & 4 & $(19 \%)$ & 7 & $(36.8 \%)$ & \\
\hline \multicolumn{8}{|l|}{ Venous invasion } \\
\hline Absent & 24 & $(60 \%)$ & 13 & $(61.9 \%)$ & 11 & $(57.9 \%)$ & \multirow{2}{*}{$0.79 \$$} \\
\hline Present & 16 & $(40 \%)$ & 8 & $(38.1 \%)$ & 8 & $(42.1 \%)$ & \\
\hline \multicolumn{8}{|l|}{ pCR } \\
\hline No & 34 & $(85 \%)$ & 21 & $(100 \%)$ & 13 & $(68.4 \%)$ & \multirow{2}{*}{$0.07 \S$} \\
\hline Yes & 6 & $(15 \%)$ & 0 & $(0 \%)$ & 6 & $(31.6 \%)$ & \\
\hline \multicolumn{8}{|l|}{ Regression group } \\
\hline 3 & 8 & $(20 \%)$ & 7 & $(33.3 \%)$ & 1 & $(5.3 \%)$ & \multirow{4}{*}{$<0.01 \S$} \\
\hline 2 & 14 & $(35 \%)$ & 13 & $(61.9 \%)$ & 1 & $(5.3 \%)$ & \\
\hline 1 & 12 & $(30 \%)$ & 1 & $(4.8 \%)$ & 11 & $(57.9 \%)$ & \\
\hline 0 & 6 & $(15 \%)$ & 0 & $(0 \%)$ & 6 & $(31.6 \%)$ & \\
\hline \multicolumn{8}{|l|}{ Regression rate } \\
\hline Lo-R & 22 & $(55 \%)$ & 20 & $(95.2 \%)$ & 2 & $(10.5 \%)$ & \multirow{2}{*}{$<0.01 \S$} \\
\hline $\mathrm{Hi}-\mathrm{R}$ & 18 & $(45 \%)$ & 1 & $(4.8 \%)$ & 17 & $(89.5 \%)$ & \\
\hline \multirow{3}{*}{ Characteristics } & & All & \multicolumn{4}{|c|}{ CD8 } & \multirow{3}{*}{$p$-value } \\
\hline & & $=40)$ & Lov & $N=18)$ & & $N=22)$ & \\
\hline & No. & (\%) & No. & (\%) & No. & $(\%)$ & \\
\hline \multicolumn{8}{|l|}{ ypT } \\
\hline урТ0 & 6 & $(15 \%)$ & 0 & $(0 \%)$ & 6 & $(27.3 \%)$ & \multirow{4}{*}{0.02} \\
\hline ypT1 & 8 & $(20 \%)$ & 1 & $(5.6 \%)$ & 7 & $(31.8 \%)$ & \\
\hline урТ2 & 15 & $(37.5 \%)$ & 8 & $(44.4 \%)$ & 7 & $(31.8 \%)$ & \\
\hline урТ3 & 11 & $(27.5 \%)$ & 9 & $(50 \%)$ & 2 & $(9.1 \%)$ & \\
\hline \multicolumn{8}{|l|}{ ypN } \\
\hline ypN0 & 21 & $(52.5 \%)$ & 8 & $(44.4 \%)$ & 13 & $(59.1 \%)$ & \multirow{3}{*}{0.36} \\
\hline ypN1 & 18 & $(45 \%)$ & 10 & $(55.6 \%)$ & 8 & $(36.4 \%)$ & \\
\hline ypN2 & 1 & $(2.5 \%)$ & 0 & $(0 \%)$ & 1 & $(4.5 \%)$ & \\
\hline \multicolumn{8}{|l|}{ Lymphatic invasion } \\
\hline Absent & 29 & $(72.5 \%)$ & 14 & $(77.8 \%)$ & 15 & $(68.2 \%)$ & \multirow{2}{*}{0.72} \\
\hline Present & 11 & $(27.5 \%)$ & 4 & $(22.2 \%)$ & 7 & $(31.8 \%)$ & \\
\hline \multicolumn{8}{|l|}{ Venous invasion } \\
\hline Absent & 24 & $(60 \%)$ & 10 & $(55.6 \%)$ & 14 & $(63.6 \%)$ & 060 \\
\hline Present & 16 & $(40 \%)$ & 8 & $(44.4 \%)$ & 8 & $(36.4 \%)$ & 0.00 \\
\hline $\mathrm{pCR}$ & & & & & & & \\
\hline No & 34 & $(85 \%)$ & 18 & $(100 \%)$ & 16 & $(72.7 \%)$ & 0.02 \\
\hline Yes & 6 & $(15 \%)$ & 0 & $(0 \%)$ & 6 & $(27.3 \%)$ & 0.02 \\
\hline Regression group & & & & & & & \\
\hline 3 & 8 & $(20 \%)$ & 6 & $(33.3 \%)$ & 2 & $(9.1 \%)$ & \\
\hline 2 & 14 & $(35 \%)$ & 12 & $(66.7 \%)$ & 2 & $(9.1 \%)$ & $<0.01$ \\
\hline 1 & 12 & $(30 \%)$ & 0 & $(0 \%)$ & 12 & $(54.5 \%)$ & $<0.01$ \\
\hline 0 & 6 & $(15 \%)$ & 0 & $(0 \%)$ & 6 & $(27.3 \%)$ & \\
\hline Regression rate & & & & & & & \\
\hline Lo-R & 22 & $(55 \%)$ & 18 & $(100 \%)$ & 4 & $(18.2 \%)$ & $<001$ \\
\hline Hi-R & 18 & (45\%) & 0 & $(0 \%)$ & 18 & $(81.8 \%)$ & 20.01 \\
\hline
\end{tabular}

Categorical variables were expressed as number (percentage; $¥$ Chi-square test for trend; $p<0.05$ is significant. 


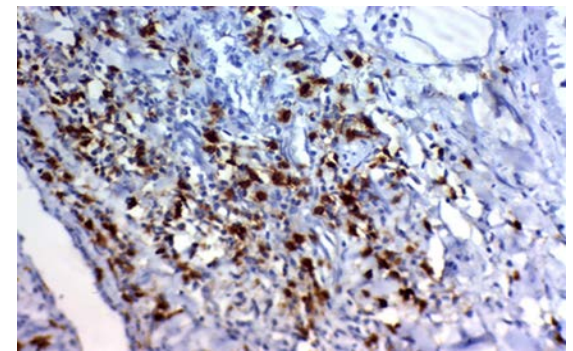

(a)

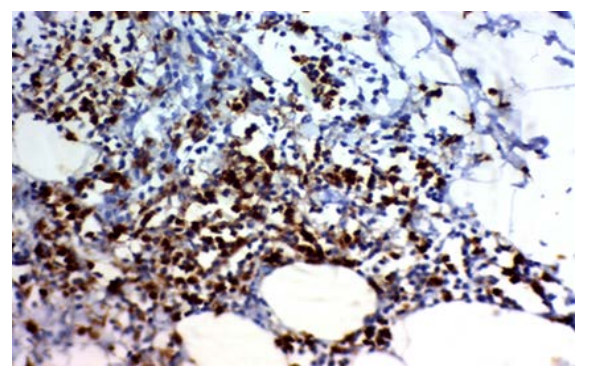

(c)

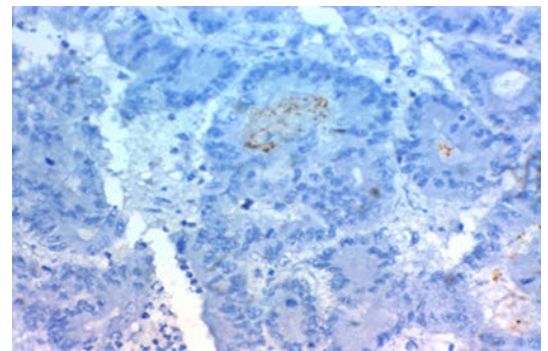

(b)

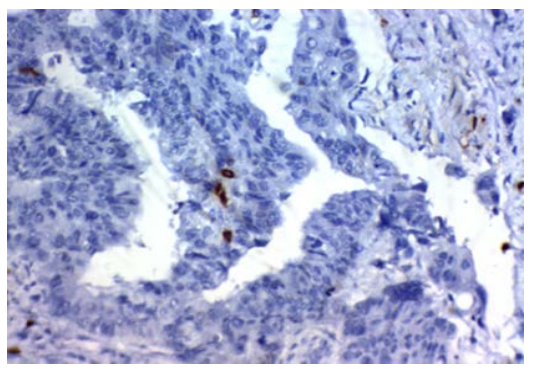

(d)

Figure 3. Immunohistochemical staining of CD8 \& CD4 in locally advanced rectal adenocarcinoma, (a) High CD4 expression in rectal adenocarcinoma Grade II; (b) Low CD4 expression in rectal adenocarcinoma Grade III; (c) High CD8 expression in rectal adenocarcinoma Grade II; (d) Low CD8 expression in rectal adenocarcinoma Grade III. ((a)-(d)) The original magnification was $\times 40$.

\section{Discussion}

Although the current multimodal treatment of locally advanced rectal cancer provided good results, we are still in need for patient-tailored treatments which expected to give greater benefit [19]. Denkert et al. [8] reported that there was significant effect for tumor infiltration of lymphocytes and achieving pCR in surgical specimens after neoadjuvant chemotherapy in breast cancer. Also a correlation between baseline peripheral lymphocyte counts and disease outcome in patients with other types of malignancies, including carcinoma of the uterine cervix and breast was reported [20].

Kitayama et al. [21] found that circulating lymphocytes play an important part for achieving benefit of preoperative radiotherapy in locally advanced rectal cancer (LARC). In our study we found that a high pre-CRT total lymphocytes count in the peripheral blood of rectal cancer patients was significantly associated with pathological response and high regression rate, and this as reported by Kitayama et al., Choi et al., and Tade et al. [21] [22] [23], but in contrary to what reported byJaesung et al. [24] who failed to find significant correlation between baseline blood lymphocyte counts with $\mathrm{pCR}$ and he explained this because they excluded patients with baseline lymphopenia from their study populations before preoperative CRT. Lymphopenia initially and lymphopenia as treatment effect has a negative prognostic value in various settings and malignancies [25] [26] [27] [28].

Flow cytometry analysis of peripheral lymphocyte subsets showed that; preCRT circulating T lymphocytes, Th lymphocytes, and Tc lymphocytes but not B lymphocytes, had positive significant correlations with the tumor regression rate 
and pathological response, and these are concordant with Tada et al., Demaria \& Formenti, and Ma et al. [23] [29] [30], as all these studies mentioned the role of T lymphocytes, but not B lymphocytes in the antitumor immune effect of radiotherapy.

It was previously demonstrated that the densities of CD4(+) and CD8(+) tumor-infiltrating lymphocytes (TIL) had significant association with the histological grade after CRT, also in achieving CR post CRT and the density of CD8(+) TIL was found as an independent prognostic factor [31]. In our results, we detected a strong correlation between the density of CD4(+) and CD8(+) T cells in biopsy samples of rectal cancer and tumor response to CRT, and this provide evidence for better response to CRT in tumor with more T lymphocytes. Favorable tumor regression was associated strongly with high number of TIL in colorectal cancer tissue [11] [31] [32] [33] [34] especially between 5-fu chemotherapy and TIL density there was positive correlation for achieving response [34]. In contrary to that, Grabenbauer et al. [6] showed that in anal squamaous cell carcinoma tumor-infiltrating CD8(+) T cells, had an adverse prognostic effect on response to CRT. This may explained by the presence of human papilloma virus which is usually associated with anal carcinoma, it affect the histological characteristics of the tumor stroma by specific viral proteins processed in tumor cells.

Studies not investigate the relation between peripheral blood lymphocytes and tumor-infiltrating lymphocytes (TIL) in wide range. Milne et al. [35] failed to document correlation between the lymphocyte count in peripheral blood and CD8(+) or CD20(+) TIL in h ovarian cancer patients, he found significant correlation of both lymphocyte count and TIL with prognosis, but the correlations were independent of each other, but in our study we are the first to find correlation between the pre CRT peripheral lymphocytes subsets and tissue expression of CD4 \& 8 in rectal cancer patients. We see significant positive correlation between pre CRT peripheral lymphocyte, T lymphocytes, Th, Tc and tissue CD4 \& CD8 and all were statistically significant correlated to tumor regression rate.

Preoperative neoadjuvant therapy is not like adjuvant therapy, because it provides other short term endpoints based on pathologic tumor response. It is now documented that after preoperative CRT for rectal cancer patients who achieving pathologic complete response (ypCR) have favorable long-term outcomes [36].

In our result, endpoints were limited to the pathologic regression but we can rely on the KROG 09-01 trial which concluded that post preoperative chemoradiotherapy in rectal cancer patients, those who gained pathological remission showed better disease outcomes. Kitayama et al. [5] showed that high lymphocyte count was associated significantly with better patients outcome regard overall and disease-free survival.

\section{Conclusion}

In conclusion, despite our study, small number of patients and short-term follow-up, we found that the pre-CRT peripheral lymphocytes count, peripheral lymphocyte subsets (T cells, Th and Tc) and tissue CD4 \& CD8 were predictors of 
pathologic tumor regression after preoperative CRT in rectal cancer patients. Also for the first time, we proved positive significant correlation between pre-CRT peripheral lymphocyte, $\mathrm{T}$ lymphocytes, $\mathrm{T}$ helper, $\mathrm{T}$ cytotoxic and high tissue lymphocyte (CD4 \& CD8). Immune response which is mediated by lymphocytes plays a positive role in the clinical response to CRT, and immune modulation through lymphocytes attractive mechanisms may help to get better effect for rectal cancer patients. Further studies with bigger patients' number and longer follow-up duration are needed.

\section{Compliance with Ethical Standards}

The authors indicated no sources of support in the form of grants, equipment or drugs.

\section{The Conflict of Interest}

The authors indicated no potential conflict of interest.

\section{References}

[1] Trakarnsanga, A., Ithimakin, S. and Weiser, M.R. (2012) Treatment of Locally Advanced Rectal Cancer: Controversies and Questions. World Journal of Gastroenterology, 18, 5521-5532.

[2] Sauer, R., Becker, H., Hohenberger, W., Rödel, C., Wittekind, C., Fietkau, R., et al. (2004) Preoperative versus Postoperative Chemoradiotherapy for Rectal Cancer. The New England Journal of Medicine, 351, 1731-1740. https://doi.org/10.1056/NEJMoa040694

[3] Yan, H., Wang, R., Zhu, K., Zhao, W. and Jiang, S. (2011) Predictors of Sensitivity to Preoperative Chemoradiotherapy of Rectal Adenocarcinoma. Tumori, 97, 717723.

[4] Kitayama, J., Yasuda, K., Kawai, K., Sunami, E. and Nagawa, H. (2011) Circulating Lymphocyte Is an Important Determinant of the Effectiveness of Preoperative Radiotherapy in Advanced Rectal Cancer. BMC Cancer, 11, 64. https://doi.org/10.1186/1471-2407-11-64

[5] Bhardwaj, N. (2007) Harnessing the Immune System to Treat Cancer. Journal of Clinical Investigation, 117, 1130-1136. https://doi.org/10.1172/JCI32136

[6] Grabenbauer, G.G., Lahmer, G., Distel, L. and Niedobitek, G. (2006) Tumor-Infiltrating Cytotoxic T Cells But Not Regulatory T Cells Predict Outcome in Anal Squamous Cell Carcinoma. Clinical Cancer Research, 12, 3355-3360. https://doi.org/10.1158/1078-0432.CCR-05-2434

[7] Kawai, O., Ishii, G., Kubota, K., Murata, Y., Naito, Y., Mizuno, T., et al. (2008) Predominant Infiltration of Macrophages and CD8(p) T Cells in Cancer Nests Is a Significant Predictor of Survival in Stage IV Nonsmall Cell Lung Cancer. Cancer, 113, 1387-1395. https://doi.org/10.1002/cncr.23712

[8] Denkert, C., Loibl, S., Noske, A., Roller, M., Muller, B.M., Komor, M., et al. (2010) Tumor-Associated Lymphocytes as an Independent Predictor of Response to Neoadjuvant Chemotherapy in Breast Cancer. Journal of Clinical Oncology, 28, 105 113. https://doi.org/10.1200/JCO.2009.23.7370

[9] Galon, J., Costes, A., Sanchez-Cabo, F., Kirilovsky, A., Mlecnik, B., Lagorce-Pages, C., et al. (2006) Type, Density, and Location of Immune Cells within Human Colo- 
rectal Tumors Predict Clinical Outcome. Science, 313, 1960-1964. https://doi.org/10.1126/science.1129139

[10] Gao, Q., Qiu, S.J., Fan, J., Zhou, J., Wang, X.Y., Xiao, Y.S., et al. (2007) Intratumoral Balance of Regulatory and Cytotoxic T Cells Is Associated with Prognosis of Hepatocellular Carcinoma after Resection. Journal of Clinical Oncology, 25, 2586-2593. https://doi.org/10.1200/JCO.2006.09.4565

[11] Ladoire, S., Martin, F. and Ghiringhelli, F. (2011) Prognostic Role of FOXP3 Regulatory T Cells Infiltrating Human Carcinomas: The Paradox of Colorectal Cancer. Cancer Immunology, Immunotherapy, 60, 909-918. https://doi.org/10.1007/s00262-011-1046-y

[12] Stipa, F., Chessin, D.B., Shia, J., Paty, P.B., Weiser, M., Temple, L.K., et al. (2006) A Pathologic Complete Response of Rectal Cancer to Preoperative Combined Modality Therapy Results in Improved Oncological Outcome Compared with Those Who Achieve No Downstaging on the Basis of Preoperative Endorectal Ultrasonography. Annals of Surgical Oncology, 13, 1047-1053. https://doi.org/10.1245/ASO.2006.03.053

[13] Uppal, S., Verma, S. and Dhot, P.S. (2003) Normal Values of CD4 and CD8 Lymphocyte Subsets in Healthy Indian Adults and the Effects of Sex, Age, Ethnicity, and Smoking. Cytometry Part B: Clinical Cytometry, 52, 32-36.

https://doi.org/10.1002/cyto.b.10011

[14] Edge, S.B. and Compton, C.C. (2010) AJCC Cancer Staging Manual. 7th Edition, Springer-Verlag, New York, 143-164.

[15] Ueno, H., Kajiwara, Y., Shimazaki, H., Shinto, E., Hashiguchi, Y., Nakanishi, K., et al. (2012) New Criteria for Histologic Grading of Colorectal Cancer. American Jour-nal of Surgical Pathology, 36, 193-201. https://doi.org/10.1097/PAS.0b013e318235edee

[16] Hsu, S.M., Raine, L. and Fanger, H. (1981) Use of Avidin-Biotin-Peroxidase Complex $(\mathrm{ABC})$ in Immunoperoxidase Techniques: A Comparison between $\mathrm{ABC}$ and Unlabeled Antibody (PAP) Procedures. Journal of Histochemistry \& Cytochemistry, 29, 577-580. https://doi.org/10.1177/29.4.6166661

[17] Shinto, E., Hashiguchi, Y., Ueno, H., Kobayashi, H., Ishiguro, M., Mochizuki, H., et al. (2011) Pretreatment CD133 and Cyclooxygenase-2 Expression as the Predictive Markers of the Pathological Effect of Chemoradiotherapy in Rectal Cancer Patients. Diseases of the Colon \& Rectum, 54, 1098-1106. https://doi.org/10.1097/DCR.0b013e3182218155

[18] Dahlin, A.M., Henriksson, M.L., Van Guelpen, B., Stenling, R., Oberg, A., Rutegard, J., et al. (2011) Colorectal Cancer Prognosis Depends on T-Cell Infiltration and Molecular Characteristics of the Tumor. Modern Pathology, 24, 671-682.

https://doi.org/10.1038/modpathol.2010.234

[19] Kalady, M.F., de Campos-Lobato, L.F., Stocchi, L., Geisler, D.P., Dietz, D., Lavery, I.C., et al. (2009) Predictive Factors of Pathologic Complete Response after Neoadjuvant Chemoradiation for Rectal Cancer. Annals of Surgery, 250, 582-589.

[20] Choi, C.H., Kang, H., Kim, W.Y., Kim, T.J., Lee, J.W., Huh, S.J., et al. (2008) Prognostic Value of Baseline Lymphocyte Count in Cervical Carcinoma Treated with Concurrent Chemoradiation. International Journal of Radiation Oncology, Biology, Physics, 71, 199-204. https://doi.org/10.1016/j.ijrobp.2007.09.024

[21] Kitayama, J., Yasuda, K., Kawai, K., Sunami, E. and Nagawa, H. (2010) Circulating Lymphocyte Number Has Positive Association with Tumor Response in Neoadjuvant Chemoradiotherapy for Advanced Rectal Cancer. Radiation Oncology, 5, 47. https://doi.org/10.1186/1748-717X-5-47 
[22] Choi, C.H., Kim, W.D., Lee, S.J. and Park, W.Y. (2012) Clinical Predictive Factors of Pathologic Tumor Response after Preoperative Chemoradiotherapy in Rectal Can-cer. Radiation Oncology, 30, 99-107 https://doi.org/10.3857/roj.2012.30.3.99

[23] Tada, N., Kazushige, K., Nelson, H., Soichiro, I. and Hironori, Y. (2015) Prediction of the Preoperative Chemoradiotherapy Response for Rectal Cancer by Peripheral Blood Lymphocyte Subsets. World Journal of Surgical Oncology, 13, 30. https://doi.org/10.1186/s12957-014-0418-0

[24] Heo, J., Chun, M., Noh, O.K., Oh, Y.T., Suh, K.W., Park, J.E. and Cho, O. (2016) Sustaining Blood Lymphocyte Count during Preoperative Chemoradiotherapy as a Pre-dictive Marker for Pathologic Complete Response in Locally Advanced Rectal Cancer. Cancer Research and Treatment, 48, 232-239.

[25] Campian, J.L., Ye, X., Brock, M. and Grossman, S.A. (2013) Treatment-Related Lym-phopenia in Patients with Stage III Non-Small-Cell Lung Cancer. Cancer Investigation, 31, 183-188. https://doi.org/10.3109/07357907.2013.767342

[26] Wild, A.T., Ye, X., Ellsworth, S.G., Smith, J.A., Narang, A.K., Garg, T., et al. (2013) The Association between Chemoradiation-Related Lymphopenia and Clinical Outcomes in Patients with Locally Advanced Pancreatic Adenocarcinoma. American Journal of Clinical Oncology, 38, 259-265. https://doi.org/10.1097/COC.0b013e3182940ff9

[27] Ray-Coquard, I., Cropet, C., Van Glabbeke, M., Sebban, C., Le Cesne, A., Judson, I., et al. (2009) Lymphopenia as a Prognostic Factor for Overall Survival in Advanced Car-cinomas, Sarcomas, and Lymphomas. Cancer Research, 69, 5383-5391. https://doi.org/10.1158/0008-5472.CAN-08-3845

[28] Grossman, S.A., Ye, X., Lesser, G., Sloan, A., Carraway, H., Desideri, S., et al. (2011) Immunosuppression in Patients with High-Grade Gliomas Treated with Radiation and Temozolomide. Clinical Cancer Research, 17, 5473-5480. https://doi.org/10.1158/1078-0432.CCR-11-0774

[29] Demaria, S. and Formenti, S.C. (2012) Role of T Lymphocytes in Tumor Response to Radiotherapy. Frontiers in Oncology, 2, 95. https://doi.org/10.3389/fonc.2012.00095

[30] Ma, Y., Kepp, O., Ghiringhelli, F., Apetoh, L., Aymeric, L., Locher, C., et al. (2010) Chemotherapy and Radiotherapy: Cryptic Anticancer Vaccines. Seminars in Immunology, 22, 113-124. https://doi.org/10.1016/j.smim.2010.03.001

[31] Yasuda, K., Nirei, T., Sunami, E., Nagawa, H. and Kitayama, J. (2011) Density of CD4(+) and CD8(+) T Lymphocytes in Biopsy Samples Can Be a Predictor of Patho-logical Response to Chemoradiotherapy (CRT) for Rectal Cancer. Radiation Oncology, 6, 49. https://doi.org/10.1186/1748-717X-6-49

[32] Koch, M., Beckhove, P., Op den Winkel, J., Autenrieth, D., Wagner, P., Nummer, D., et al. (2006) Tumor Infiltrating T Lymphocytes in Colorectal Cancer: TumorSelective Activation and Cytotoxic Activity in Situ. Annals of Surgery, 244, 986-992.

[33] Morris, M., Platell, C. and Iacopetta, B. (2008) Tumor-Infiltrating Lymphocytes and Per-foration in Colon Cancer Predict Positive Response to 5-Fluorouracil Chemotherapy. Clinical Cancer Research, 14, 1413-1417. https://doi.org/10.1158/1078-0432.CCR-07-1994

[34] Laghi, L., Bianchi, P., Miranda, E., Balladore, E., Pacetti, V., Grizzi, F., et al. (2009) $\mathrm{CD}^{3+}$ Cells at the Invasive Margin of Deeply Invading (pT3-T4) Colorectal Cancer and Risk of Post-Surgical Metastasis: A Longitudinal Study. The Lancet Oncology, 10, 877-884. https://doi.org/10.1016/S1470-2045(09)70186-X

[35] Milne, K., Alexander, C., Webb, J.R., Sun, W., Dillon, K., Kalloger, S.E., et al. (2012) Absolute Lymphocyte Count Is Associated with Survival in Ovarian Cancer Inde- 
pendent of Tumor-Infiltrating Lymphocytes. Journal of Translational Medicine, 10, 33. https://doi.org/10.1186/1479-5876-10-33

[36] Yeo, S.G., Kim, D.Y., Kim, T.H., Chang, H.J., Oh, J.H., Park, W., et al. (2010) Pathologic Complete Response of Primary Tumor Following Preoperative Chemoradiotherapy for Locally Advanced Rectal Cancer: Long-Term Outcomes and Prognostic Sig-nificance of Pathologic Nodal Status (KROG 09-01). Annals of Surgery, 252, 998-1004. https://doi.org/10.1097/SLA.0b013e3181f3f1b1

Submit or recommend next manuscript to SCIRP and we will provide best service for you:

Accepting pre-submission inquiries through Email, Facebook, LinkedIn, Twitter, etc. A wide selection of journals (inclusive of 9 subjects, more than 200 journals)

Providing 24-hour high-quality service

User-friendly online submission system

Fair and swift peer-review system

Efficient typesetting and proofreading procedure

Display of the result of downloads and visits, as well as the number of cited articles Maximum dissemination of your research work

Submit your manuscript at: http://papersubmission.scirp.org/

Orcontact jct@scirp.org 
